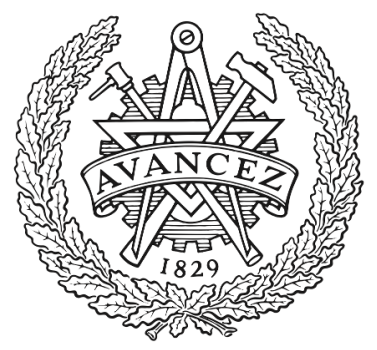

CHALMERS

UNIVERSITY OF TECHNOLOGY

\title{
Simultaneous Search for Prompt Radio Emission Associated with the Short GRB 170112A Using the All-sky Imaging Capability of the OVRO-LWA
}

Downloaded from: https://research.chalmers.se, 2023-04-26 11:56 UTC

Citation for the original published paper (version of record):

Anderson, M., Hallinan, G., Eastwood, M. et al (2018). Simultaneous Search for Prompt Radio Emission Associated with the Short GRB 170112A Using the All-sky Imaging Capability of the OVRO-LWA. Astrophysical Journal, 864(1).

http://dx.doi.org/10.3847/1538-4357/aad2d7

N.B. When citing this work, cite the original published paper. 


\title{
A Simultaneous Search for Prompt Radio Emission Associated with the Short GRB 170112A Using the All-sky Imaging Capability of the OVRO-LWA
}

\author{
Marin M. Anderson ${ }^{1}$, Gregg Hallinan ${ }^{1}$, Michael W. Eastwood ${ }^{1}$ (D), Ryan M. Monroe ${ }^{1}$, Harish K. Vedantham ${ }^{1}$ (D), Stephen Bourke ${ }^{1,2}$, \\ Lincoln J. Greenhill ${ }^{3}$, Jonathon Kocz ${ }^{1}$, T. Joseph W. Lazio ${ }^{4}$, Danny C. Price ${ }^{3,5}$ (i), Frank K. Schinzel ${ }^{6,7}$ (iD, \\ Yuankun Wang ${ }^{1}$, and David P. Woody ${ }^{8}$ \\ ${ }^{1}$ California Institute of Technology, 1200 E California Boulevard MC 249-17, Pasadena, CA 91125, USA; mmanders@astro.caltech.edu \\ ${ }^{2}$ Department of Space, Earth and Environment, Chalmers University of Technology, Onsala Space Observatory, SE-439 92 Onsala, Sweden \\ ${ }^{3}$ Harvard-Smithsonian Center for Astrophysics, 60 Garden Street, Cambridge MA 02138, USA \\ ${ }^{4}$ Jet Propulsion Laboratory, California Institute of Technology, 4800 Oak Grove Drive, Pasadena, CA 91109, USA \\ ${ }^{5}$ Centre for Astrophysics \& Supercomputing, Swinburne University of Technology, P.O. Box 218, Hawthorn, VIC 3122, Australia \\ ${ }^{6}$ National Radio Astronomy Observatory, P.O. Box O, Socorro, NM 87801, USA \\ ${ }^{7}$ Department of Physics and Astronomy, University of New Mexico, Albuquerque, NM 87131, USA \\ ${ }^{8}$ California Institute of Technology, Owens Valley Radio Observatory, Big Pine, CA 93513, USA \\ Received 2017 November 17; revised 2018 July 1; accepted 2018 July 9; published 2018 August 27
}

\begin{abstract}
We have conducted the most sensitive low-frequency (below $100 \mathrm{MHz}$ ) search to date for prompt, low-frequency radio emission associated with short-duration gamma-ray bursts (GRBs), using the Owens Valley Radio Observatory Long Wavelength Array (OVRO-LWA). The OVRO-LWA's nearly full-hemisphere field of view ( $\sim 20,000$ square degrees) allows us to search for low-frequency (sub-100 MHz) counterparts for a large sample of the subset of GRB events for which prompt radio emission has been predicted. Following the detection of short GRB 170112A by Swift, we used all-sky OVRO-LWA images spanning one hour prior to and two hours following the GRB event to search for a transient source coincident with the position of GRB 170112A. We detect no transient source to within a $3 \sigma$ flux density limit of $4.5 \mathrm{Jy}$ at $13 \mathrm{~s}$ timescales for frequencies spanning $27-84 \mathrm{MHz}$. We place constraints on a number of models predicting prompt, low-frequency radio emission accompanying short GRBs and their potential binary neutron star merger progenitors, and place an upper limit of $L_{\text {radio }}$ $L_{\gamma} \lesssim 3.5 \times 10^{-6}$ on the fraction of energy released in the prompt radio emission, under the assumptions of negligible scattering of the radio pulse and beaming of emission along the line of sight. These observations serve as a pilot effort for a program targeting a wider sample of both short and long GRBs with the OVRO-LWA, including bursts with confirmed redshift measurements that are critical to placing constraining limits on prompt radio emission models, as well as a program for the follow-up of gravitational wave compact binary coalescence events detected by advanced LIGO and Virgo.
\end{abstract}

Key words: gamma-ray burst: general - gamma-ray burst: individual (170112A) - gravitational waves - radiation mechanisms: non-thermal - radio continuum: general

\section{Introduction}

The detection of the first gamma-ray bursts (GRBs) in 1967 heralded a race to better characterize, classify, and identify the nature of the progenitors of these seconds-long bursts of $\mathrm{MeV}$ gamma-rays, which appeared to be isotropically distributed across the sky and thus likely of cosmic origin (Klebesadel et al. 1973). Systematic detections of GRBs over the following decades revealed two distinct classes of events: the spectrally hard, short (typical duration $<2 \mathrm{~s}$ ) GRBs and the spectrally soft, long (typical duration $>2$ s) GRBs (Kouveliotou et al. 1993). The rapid followup capabilities of the BATSE instrument and BeppoSAX led to the detection of X-ray, optical, and, later, radio afterglows that provided critical information regarding distance, host galaxy association, isotropic energy estimates, source size evolution, and insight into GRB progenitors (Costa et al. 1997; Frail et al. 1997; van Paradijs et al. 1997). Routine follow-up and afterglow detection revealed that the distinct phenomenology of short and long GRBs also reflects distinct progenitor systems. Long GRB hosts are exclusively star-forming galaxies (Savaglio et al. 2009), and the location of long GRBs within their host galaxies correlates strongly with ultraviolet light, implying that long GRBs trace regions of active massive star formation (Fruchter et al. 2006). This, combined with the association of long GRBs with Type Ic core-collapse supernovae ( $\mathrm{SNe}$ ), points to massive stars as the progenitors of long GRBs (Woosley \& Bloom 2006).

The progenitors of short GRBs, however, have remained more elusive. While significant evidence exists for the association of short GRBs with compact object mergers consisting of neutron star binaries (NS-NS) or neutron star black hole binaries (NS-BH; Narayan et al. 1992), the association is not as definitive as that of long GRBs with core-collapse supernovae (see, e.g., Lyutikov 2009). However, the compact object merger scenario is the favorable progenitor model for short GRBs for the following reasons: (1) short GRBs are found in both early- and late-type galaxies, consistent with the formation of progenitor binary systems following a delay-time distribution and therefore being found in both young and old stellar populations; (2) localization within the host galaxy provided by detections of short GRB afterglows indicates a population distribution with significantly larger host galaxy offset relative to the long GRB/core-collapse SN population, as expected for a compact object binary progenitor born with a natal kick (Bloom et al. 1999; Belczynski et al. 2006); and (3) unlike long GRBs, short GRBs have no established association with supernovae (Berger et al. 2005; Bloom et al. 2006; Soderberg et al. 2006; Berger 2009), although they are associated with kilonovae/macronovae believed to be powered by 
Table 1

Models for Prompt Radio Counterparts to GRBs

\begin{tabular}{|c|c|c|c|c|c|c|}
\hline (1) & (2) & (3) & (4) & (5) & (6) & (7) \\
\hline Reference(s) & Delay Relative to GRB & $t_{\text {Intrinsic }, R}$ & $\begin{array}{l}\text { Flux Density } \\
(\mathrm{Jy})\end{array}$ & Spectral Index & $\begin{array}{l}\text { B-field } \\
\text { (G) }\end{array}$ & $L_{R} / L_{\gamma}$ \\
\hline Hansen \& Lyutikov (2001)/Lyutikov (2013) a & $10-100 \mathrm{~ms}$ before & $\sim 4 \mathrm{~ms}$ & 0.09 & -1 & $10^{12}$ & $10^{-10} \propto \epsilon$ \\
\hline Usov \& Katz $(2000)^{c}$ & simultaneous & $t_{\gamma, \text { prompt }}$ & 350 & -0.6 & $10^{15}-10^{16}$ & $10^{-4} \equiv \delta$ \\
\hline
\end{tabular}

Notes. The estimates and parameters used to calculate the predicted flux density $S$ use the approximate values reported in the respective model papers as astrophysically plausible and/or most-likely values. We emphasize that many of the parameters estimated in these models are unknown and not constrained astrophysically; therefore, the appropriate ranges for these values can vary by orders of magnitude. (1) Reference for model predicting coherent radio emission associated with GRB. (2) Onset of radio emission relative to onset of gamma-ray emission. (3) Duration of coherent radio emission, before dispersion. (4) Modelpredicted flux density for a burst located at $D=1 \mathrm{Gpc}$, the approximate distance upper limit to GRB 170112A. (5) Spectral index. (6) Magnetic field strengths required by the model in the GRB progenitor in order to satisfy conditions necessary for producing coherent radio emission. (7) Luminosity ratio between energy released in radio frequencies to energy released in gamma-rays, during the prompt emission stage. See Palmer (1993).

${ }^{a}$ Coherent radio emission is generated in the pre-merger phase through the magnetospheric interactions between the two components of the binary system; the resulting magnetized wind produces coherent radio emission with luminosity that scales as $t^{-1 / 4}$ until it peaks at the time of merger, which is estimated to occur between 10 and $100 \mathrm{~ms}$ prior to the production of the GRB. Flux density prediction in column (4) is calculated at $30 \mathrm{MHz}$ for the $\sim 4 \mathrm{~ms}$ period during which the emission peaks as the neutron stars make contact and finally merge. The flux density prediction also assumes that the efficiency with which wind power is converted into coherent radio emission is $\epsilon_{R}=10^{-5}$. See Equation (13) in Lyutikov (2013).

${ }^{\mathrm{b}}$ Coherent radio emission is generated by the rapid conversion of rotational energy into magnetic energy in the rotationally supported massive NS, which forms following the merger of the BNS system, but prior to its final collapse and the generation of the GRB. The flux density prediction in column (4) is calculated at $30 \mathrm{MHz}$, and assumes a conversion coefficient $\eta$, defined by the authors as the efficiency with which spin-down energy is converted into radio luminosity, as $\eta=10^{-5}$. See Equation (7) in Pshirkov \& Postnov (2010).

${ }^{\mathrm{c}}$ Coherent radio emission occurs simultaneously to gamma-ray emission and is powered by Langmuir waves generated at the boundary between the highly magnetized outflow and the surrounding ambient medium of the GRB. The flux density prediction in column (4) is calculated at $30 \mathrm{MHz}$, for a burst like GRB $170112 \mathrm{~A}$ with duration $0.06 \mathrm{~s}$ and fluence $0.13 \times 10^{-7} \mathrm{erg} \mathrm{cm}^{-2}$, and assumes $\delta$, defined by the authors as the fluence in radio emission relative to the fluence measured in gamma-rays, as $\delta=10^{-4}$. See Equation (14) in Usov \& Katz (2000).

r-process nucleosynthesis in the expanding post-merger ejecta (Li \& Paczyński 1998; Tanvir et al. 2013; Yang et al. 2015).

A number of models predict a highly speculative but potentially very valuable counterpart to GRBs and NS-NS (BH) mergers in the form of a short, bright, coherent pulse of low-frequency radio emission (Table 1). The models predicting this coherent radio emission span all stages of the compact object merger process, from (1) the final inspiral of the binary neutron stars, to (2) a short-lived, postmerger supramassive neutron star, to (3) the post-collapse stage during which the gamma-ray emission is produced. Hansen \& Lyutikov (2001) consider the magnetospheric interaction of an NS-magnetar binary system and the generation of a coherent radio burst in the surrounding plasma environment during the pre-merger (1) phase (see also Lyutikov 2013). Pshirkov \& Postnov (2010) consider a low-frequency radio burst generated in the relativistic plasma outflow from the highly magnetized, rapidly rotating magnetar, which is predicted to form in the brief stage (2) between the merger and final collapse. Usov \& Katz (2000) predict a low-frequency radio burst that may be produced in the post-merger (3) phase (as well as in long GRBs) through the interaction of a strongly magnetized wind with the circumburst medium. In this model, the coherent lowfrequency emission is produced by the time-variable surface current that exists at the wind/ambient plasma boundary. Other models predicting coherent radio emission postmerger include synchrotron maser emission generated during the GRB fireball phase (Sagiv \& Waxman 2002), and inverse Compton radiation generated in the surrounding magnetized plasma by magnetohydrodynamic modes excited by the gravitational waves (GWs) produced in the merger (Moortgat \& Kuijpers 2005).

Despite the diversity of models, common to all is the prediction that a GRB is accompanied by a coherent and intrinsically shortduration burst of radio emission that occurs within a window of several seconds to the production of the gamma-ray emission, with a steep negative spectral index that favors observations at lower frequencies. In the case of Usov \& Katz (2000), the emission is predicted to peak at $\sim \mathrm{MHz}$ frequencies and fall off rapidly above roughly $30 \mathrm{MHz}$. Many of the models also require the presence of extreme, magnetar-strength magnetic fields (e.g., as high as $10^{15} \mathrm{G}$ ) - atypical given the expected age of these systems at the time of merger (Goldreich \& Reisenegger 1992), but justified in the models through magnetic field amplification during the coalescence of the system (and through numerical simulations, e.g., Duez et al. 2006). Magnetic field amplification has also been predicted for post-merger hypermassive neutron star remnants and invoked to explain short GRB extended emission (Metzger et al. 2008) and the kilonova emission of GW1701817 (Metzger et al. 2018).

Searches for prompt, coherent radio counterparts to GRBs are made difficult by the need for observations that satisfy the requirements for high sensitivity at sufficiently low frequency, and are coincident with (or dependent on the amount of dispersive delay shortly after) the detection of the corresponding GRB. There have been many searches for prompt, coherent radio counterparts to GRBs to date, but none have yielded detections thus far (e.g., Baird et al. 1975; Inzani et al. 1982; Koranyi et al. 1995; Dessenne et al. 1996; Benz \& Paesold 1998; Balsano 1999; Bannister et al. 2012, see Granot \& van der Horst 2014 for brief summary). Most recently, Obenberger et al. (2014) searched for 
prompt emission from 32 GRBs using the LWA1 Prototype All Sky Imager (PASI; Ellingson et al. 2013; Obenberger et al. 2015), and Kaplan et al. (2015) conducted follow-up observations of the short GRB 150424A, starting within 23 s of the detected gammarays, with the Murchison Widefield Array (MWA; Tingay et al. 2013) at frequencies above $80 \mathrm{MHz}$. See Table 2 for a summary of previous radio surveys specifically targeting prompt emission associated with GRBs.

Despite these difficult observational requirements, and the speculative nature of the models, low-frequency radio searches for counterparts to short GRBs remain valuable. Systematic detection would provide a radio source population with the ability to probe the density and turbulence of the intergalactic medium(IGM; Inoue 2004), with utility as a diagnostic of accretion-powered jet physics (Macquart 2007), and as a valuable electromagnetic (EM) counterpart for radio follow-up of GW events (e.g., Kaplan et al. 2016). Most critically, the detection of a radio pulse associated with a short GRB would provide independent confirmation of the association of short GRBs with neutron star mergers. This is especially relevant in the current era of multimessenger astronomy ushered in by the detection of GW170817 (Abbott et al. 2017d), which provided the long sought-after first direct association between GRBs and binary neutron star mergers. However, the GRB detected in association with GW170817 remains distinct from the "classical" short GRBs that are systematically detected at larger distances (Abbott et al. 2017a). A coherent, low-frequency radio counterpart to binary neutron star mergers would provide a direct link between short GRBs and their more local GW-detected counterparts.

We have conducted the most sensitive search to date at frequencies below $100 \mathrm{MHz}$ for a prompt, coherent radio counterpart associated with GRBs, using the Owens Valley Radio Observatory Long Wavelength Array (OVRO-LWA) to observe the field of the short GRB 170112A. The lowfrequency $(27-85 \mathrm{MHz})$ and simultaneous nature of our observations provides constraining limits on a number of the prompt radio emission counterpart models. In Section 2, we describe the OVRO-LWA and our observations of GRB 170112A. In Section 3, we describe the analysis, including our dedispersion search. In Section 4, we place limits on any prompt radio emission associated with $170112 \mathrm{~A}$ and the resulting constraints on the models, as well the relevance of the OVRO-LWA observations in the context of GW follow-up. We conclude in Section 5.

\section{Observations}

\subsection{OVRO-LWA}

The OVRO-LWA is a 352-element, dual-polarization dipole array currently under development at the Owens Valley Radio Observatory (OVRO) in Owens Valley, California (G. Hallinan et al. 2018, in preparation), operating at frequencies below $100 \mathrm{MHz}$. The final array will be spread over a $2.5 \mathrm{~km}$ diameter area, providing a roughly 5 arcmin spatial resolution. Full crosscorrelation of all 352 elements will enable imaging of the entire viewable sky, with a cadence of a few seconds and $100 \mathrm{mJy}$ snapshot sensitivity. Early science observations have commenced on OVRO-LWA, with key science including low-frequency radio transients, exo-space weather monitoring of nearby stellar systems, cosmic dawn $21 \mathrm{~cm}$ science (see Eastwood et al. 2018; Price et al. 2018), ionospheric studies, solar dynamic imaging spectroscopy, and monitoring of the Jovian system. A unique design feature of the array is the nonconflicting nature of these disparate science goals, which all share a common mode of observing and initial data products, meaning that all science objectives can be served simultaneously.

The current, stage II OVRO-LWA, which incorporates 256 elements from the $200 \mathrm{~m}$ diameter core and the 32element Long Baseline Demonstrator Array (LBDA), has been observing continuously since 2016 December, operating from 27 to $84 \mathrm{MHz}$ (2400 channels) with a $13 \mathrm{~s}$ cadence. Full cross-correlation of 512 inputs (256 antennas $\times 2$ polarizations) by the Large-Aperture Experiment to Detect the Dark Age (LEDA) correlator (Kocz et al. 2015) provides a full-sky field of view with an approximately 10 arcmin resolution at the top of the observing band. Data are continuously written to a multiday buffer. Data corresponding to triggers of interest are copied to the on-site AllSky Transient Monitor for storage and processing. Example triggers of interest include Swift and Fermi alerts for both short and long GRBs as well as GW LIGO-Virgo Collaboration events, as distributed by the Gamma-ray Coordinates Network. ${ }^{9}$ The short GRB 170112A represents the first short GRB with sufficient position localization to search for a coherent radio emission counterpart with the OVRO-LWA, following the onset of our stage II continuous mode of observing. The results of follow-up observations of a larger sample of both long and short GRBs with known redshift will be released once the stage II OVRO-LWA continuous operations have completed.

\subsection{Short GRB $170112 A$}

The short GRB 170112A was detected on 2017 January 12 02:02:00 UTC by the Swift Burst Alert Telescope (BAT; Gehrels et al. 2004; Krimm et al. 2013) at the position (R.A., decl. $)=\left(01^{\mathrm{h}} 00^{\mathrm{m}} 55^{\mathrm{s}} .7,-17^{\circ} 13^{\prime} 57\right.$ !' 9$)$, to within a $90 \%$ error region of radius $2.5 \mathrm{arcmin}$ (Lien et al. 2017; Mingo et al. 2017). The burst was identified as a hard, short burst (power-law index $\alpha_{\mathrm{PL}}=-1.2$ and burst fluence $S=0.13 \times 10^{-7} \mathrm{erg} \mathrm{cm}^{-2}$ in the Swift-BAT $15-150 \mathrm{keV}$ band), with an atypically short duration of $T_{90}=0.06 \mathrm{~s}$ (Lien et al. 2017, see the discussion in Section 3.1). No extended emission was found, as is typical of the majority of short GRBs (Bostanciet al. 2013). Due to the lack of detected X-ray flare or afterglow emission, by either the Swift X-Ray Telescope (D'Ai et al. 2017) or Swift Ultraviolet/Optical Telescope (Siegel \& Mingo 2017), following the initial BAT trigger, the position of 170112A is known only to within the 2.5 arcmin position as measured by BAT. No afterglow counterpart or associated host galaxy was found in the observations conducted by follow-up ground-based optical and NIR facilities. D'Avanzo et al. (2017) observed the location of GRB 170112A with the REM $60 \mathrm{~cm}$ robotic telescope at La Silla Observatory in Chile and placed an 18.0 mag $\mathrm{H}$ band upper limit on an afterglow counterpart. Mazaeva et al. (2017) report the detection by the $0.7 \mathrm{~m}$ AS-32 telescope at Abastumani Observatory of a source, inside the BAT error region, which is not present in the USNO-B1.0 catalog but is detected in the DSS2 (red) survey. We therefore disregard the association of this source with GRB 170112A, and in the remaining analysis consider GRB 170112A as a short burst with neither detected afterglow emission nor associated host galaxy.

The OVRO-LWA was observing simultaneously to the detection of 170112 A by Swift-BAT. Following the GCN

\footnotetext{
9 GCN: https://gcn.gsfc.nasa.gov/.
} 
Table 2

Searches Targeting Prompt, Coherent Radio Emission Associated with GRBs

\begin{tabular}{|c|c|c|c|c|c|c|c|c|}
\hline (1) & $(2)$ & (3) & (4) & (5) $\ldots$ & $(6)$ & (7) & $(8)$ & (9) \\
\hline Reference(s) & Instrument & $\begin{array}{l}\text { Frequency } \\
(\mathrm{MHz})\end{array}$ & $\begin{array}{l}\text { Bandwidth } \\
\text { (MHz) }\end{array}$ & $\begin{array}{l}\text { Sensitivity } \\
\text { (Jy) }\end{array}$ & $\begin{array}{l}\text { Time res. } \\
\text { (s) }\end{array}$ & $\begin{array}{c}\text { Frequency res. } \\
(\mathrm{MHz})\end{array}$ & $t_{\text {on sky }}$ & $N_{\mathrm{GRBs}}$ \\
\hline$\overline{\text { Baird et al. (1975) }}$ & spaced receivers $^{\mathrm{a}}$ & 151 & 1 & $10^{5}$ & 0.3 & 0.2 & $-1 \mathrm{hr}$ & 19 \\
\hline Cortiglioni et al. (1981) & VHF/UHF station at Medicina & 151,408 & 1,2 & $10^{4}$ & 0.3 & $\ldots$ & $-1 \mathrm{hr}$ & 32 \\
\hline Inzani et al. (1982) & VHF/UHF station at Medicina & 151,408 & 1 & $10^{4}$ & 1 & $\ldots$ & $-1 \mathrm{hr}$ & 65 \\
\hline Koranyi et al. (1995) & CLFST & 151 & $\cdots$ & 200 & 1.5 & $\cdots$ & $1 \mathrm{hr}$ & 1 \\
\hline Dessenne et al. (1996) & CLFST & 151 & 0.7 & $73,35,15$ & 1.5 & $\ldots$ & $-280,6,16 \mathrm{~m}$ & 2 \\
\hline Benz \& Paesold (1998) & solar radio spectrometers ${ }^{\mathrm{b}}$ & $40-1000$ & $\ldots$ & $10^{5}$ & 0.25 & 1 & simultaneous & 7 \\
\hline Balsano (1999) & FLIRT & 74 & 1.9 & $10^{3}$ & 0.05 & $\ldots$ & $10 \mathrm{~s}$ & 32 \\
\hline Bannister et al. (2012) & 12-m dish at Parkes Observatory & 1400 & 220 & 7 & $64 \times 10^{-6}$ & 0.390 & $200 \mathrm{~s}$ & 9 \\
\hline Obenberger et al. (2014) & LWA1 & $37.9,52,74$ & 0.075 & $68,65,70$ & 5 & 0.0167 & simultaneous & 34 \\
\hline Kaplan et al. (2015) & MWA & $80,88.9,97.9,108.1,119.7,132.5$ & 2.56 & $8.7,7.7,5.7,4.9,4.2,3.0$ & 4 & $\cdots$ & $23 \mathrm{~s}$ & 1 \\
\hline This Work & OVRO-LWA & 56 & 57 & 4.5 & 13 & 0.024 & $-1 \mathrm{hr}$ & 1 \\
\hline
\end{tabular}

Notes. (8) Start of observations, relative to the time of GRB. (9) Number of GRBs targeted in the radio observations.

a The receivers were located in stations at Cambridge, UK; Dublin, Ireland; Glasgow, UK; Harwell, UK; Jodrell Bank, UK; and Malta

${ }^{\mathrm{b}}$ Located in Bleien Radio Observatory, Switzerland; Tremsdorf, Germany; and Weissenau Observatory, Germany. 
detection notice, and after receiving the verification by the GCN circular (Mingo et al. 2017) that Swift had detected a short GRB, we saved three hours of data from the OVROLWA transient buffer, corresponding to one hour prior to and two hours after the Swift detection (UT range 01:02:02-04:02:18). The data consist of 832 contiguous integrations of $13 \mathrm{~s}$ duration, with 2398 frequency channels spanning $27.38-84.92 \mathrm{MHz}(24 \mathrm{kHz}$ frequency resolution). The OVRO-LWA is a zenith-pointing telescope, and $170112 \mathrm{~A}$ was located at an elevation of approximately $35^{\circ}$ in the primary beam.

The visibility data delivered from the LEDA correlator are converted from their raw format into the standard Common Astronomy Software Applications (CASA; McMullin et al. 2007) visibility table measurement sets. The data are then flagged in frequency, antenna, and baseline space, using a combination of manual inspection and an automated in-house algorithm that fits for smoothness in the visibilities across frequency, time, and $u v w$-space (R. M. Monroe et al. 2018, in preparation). Roughly 60 antennas are flagged for the duration of the observation, the majority of these due to issues along the signal path resulting in a loss of sky signal from a subset of antennas. Less than $10 \%$ of the frequency band is flagged for radio frequency interference (RFI). The result is that approximately $45 \%$ of visibilities are flagged per integration. The data are calibrated using a simplified sky model consisting of the two brightest sources in the sub- $100 \mathrm{MHz}$ sky: the radio galaxy Cygnus (Cyg) A and the supernova remnant Cassiopeia (Cas) A. The complex (amplitude and phase) antenna gains are determined on a per-channel basis from the Cyg A-Cas A sky model using the CASA bandpass task. The calibration solutions are derived from an integration taken at 2017 January 11 20:26 UT, $4 \mathrm{hr}$ prior to the start of the GRB follow-up observations, when Cyg A is at its highest elevation in the beam $\left(\sim 87^{\circ}\right)$. The flux scale and bandpass are corrected by modifying the antenna gain amplitudes to fix the spectrum of Cyg A to that of Baars et al. (1977). Direction-dependent calibration in the directions of Cyg A and Cas A is performed using the TTCal calibration software package developed for the OVRO-LWA (Eastwood 2016). This is necessary in order to accurately peel the sources from the visibilities and avoid sidelobe contamination in the images, caused by antenna gain pattern variations between individual dipoles. Imaging and deconvolution are performed with WSClean (Offringa et al. 2014a, 2014b). The full field of view is imaged over $4096 \times 4096$ pixels, with a pixel scale of 1!875 and using a robust visibility weighting of 0 (Briggs 1995). At the time of these observations, a set of northeast LBDA antennas were not operational, resulting in an abnormally elongated synthesized beam with a major axis of $29^{\prime}$, a minor axis of 13.5 , and a position angle of $50^{\circ}$.

Figure 1 shows the $13 \mathrm{~s}$, full-band snapshot OVRO-LWA image corresponding to the time of the GRB detection by Swift. The images are confusion-noise limited to $\sim 800 \mathrm{mJy}$ at zenith. The image cutout in Figure 1 shows the $10^{\circ} \times 10^{\circ}$ region surrounding the position of $170112 \mathrm{~A}$, including two nearby VLA Low Frequency Sky Survey (VLSS; Lane et al. 2014) sources, VLSS J0108.2-1604 (3C 032), and VLSS J0102.6-2152. These were used to verify the flux scale at this part of the primary beam and track the position offset of sources over the course of the observation caused by ionospheric refraction. An ionospheric substorm can be seen moving through this region of the sky, particularly during the first hour of observations; however, the maximum source position offsets are within the size of the synthesized beam, and on average are of the order of a few arcminutes.

\section{Analysis}

To search for the presence of a low-frequency counterpart to $170112 \mathrm{~A}$ in our data, we searched the flux density time series at the position of $170112 \mathrm{~A}$, known to within one synthesized beam, for statistically significant peaks indicative of a radio burst on our shortest, $13 \mathrm{~s}$ integration timescales. In each integration, the median flux of an annulus around the GRB position of width $6 \pm 1$ synthesized beams was subtracted from the flux measured at the pixel corresponding to the position of GRB 170112A in order to remove additional flux from any large-scale, diffuse structure. This was done for the full $57 \mathrm{MHz}$ bandwidth, as well as the bottom (centered at $37 \mathrm{MHz})$, middle $(56 \mathrm{MHz})$, and top $(75 \mathrm{MHz})$ thirds of the band. Figure 2 shows the dedispersed time series for these four bands for the duration of the three-hour observation, for a dispersion measure (DM) of $260 \mathrm{pc} \mathrm{cm}^{-3}$ (see Section 3.1). We find no statistically significant peaks at $13 \mathrm{~s}$ timescales in any of the four bands, with typical noise in the full band, bottom band, middle band, and top band light curves of $1.5 \mathrm{Jy}, 3.6 \mathrm{Jy}, 2.2 \mathrm{Jy}$, and $1.9 \mathrm{Jy}$, respectively.

\subsection{Pulse Propagation: Dispersion and Scattering}

The propagation of a coherent radio pulse associated with $170112 \mathrm{~A}$ would be affected by the cold plasma in the intervening medium between the birth site of the pulse and the Earth. The signal will be dispersed, with lower frequencies arriving at later times relative to higher frequencies, following $t_{\text {arrival }}=4.2 \mathrm{DM} \nu_{\mathrm{GHz}}^{-2} \mathrm{~ms}$ (Cordes \& McLaughlin 2003). The amount of dispersion, quantified by the DM, is determined by the column density of electrons along the pulse path (density of electrons integrated along the line of sight), and is equal to $\mathrm{DM}=\int_{0}^{D} n_{e}(l) d l$, where $D$ is the distance to the source and $n_{e}$ is the density of electrons. The DM can be approximated as the sum of DM contributions from the Milky Way $\left(\mathrm{DM}_{\mathrm{MW}}\right)$, the intergalactic medium (DM $\mathrm{DGM}_{\mathrm{IGM}}$, and the host galaxy of the burst $\left(\mathrm{DM}_{\text {host }}\right)$. The $\mathrm{DM}$ contribution from the Milky Way is minimal due to the location of 170112A at high Galactic latitude $\left(l=135^{\circ} .9, b=-79^{\circ} .9\right) ; \mathrm{DM}_{\mathrm{MW}} \sim 30 \mathrm{pc} \mathrm{cm}^{-3}$ based on the NE2001 model of Cordes \& Lazio (2002). ${ }^{10} \mathrm{We}$ assume that the contributions from the host galaxy and circumburst environment of 170112A are similarly small, given the likely binary NS-NS(BH) progenitors of short GRBs and the typically large ( $4.5 \mathrm{kpc}$ projected median) offsets from the center of their hosts galaxies (Fong \& Berger 2013). We assume a $\mathrm{DM}_{\text {host }} \approx \mathrm{DM}_{\mathrm{MW}}$. The $\mathrm{DM}$ contribution from the IGM can be estimated as $\mathrm{DM}_{\mathrm{IGM}} \sim 1000 \mathrm{z} \mathrm{pc} \mathrm{cm}^{-3}$ (Zheng et al. 2014). Because 170112A has no optical afterglow detection and no identified host galaxy, and thus is of unknown redshift, $\mathrm{DM}_{\mathrm{IGM}}$ is highly uncertain. However, we exploit the relationship between redshift and minimum burst duration of the prompt gamma-ray emission $\left(T_{90}\right)$ determined using the sample of Swift-detected GRBs with redshift identifications from the third Swift-BAT GRB catalog (Lien et al. 2016) to place an upper limit on the redshift of 170112A of $z \lesssim 0.2$. The correlation between minimum detectable $T_{90}$ and redshift found

\footnotetext{
${ }^{10}$ https://www.nrl.navy.mil/rsd/RORF/ne2001/
} 


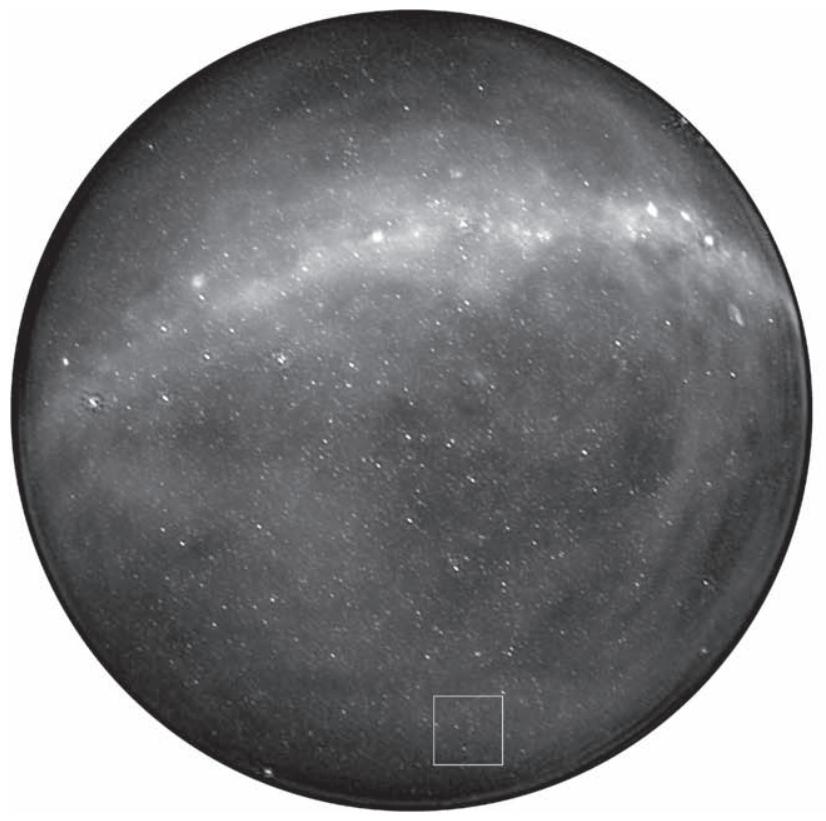

(a)

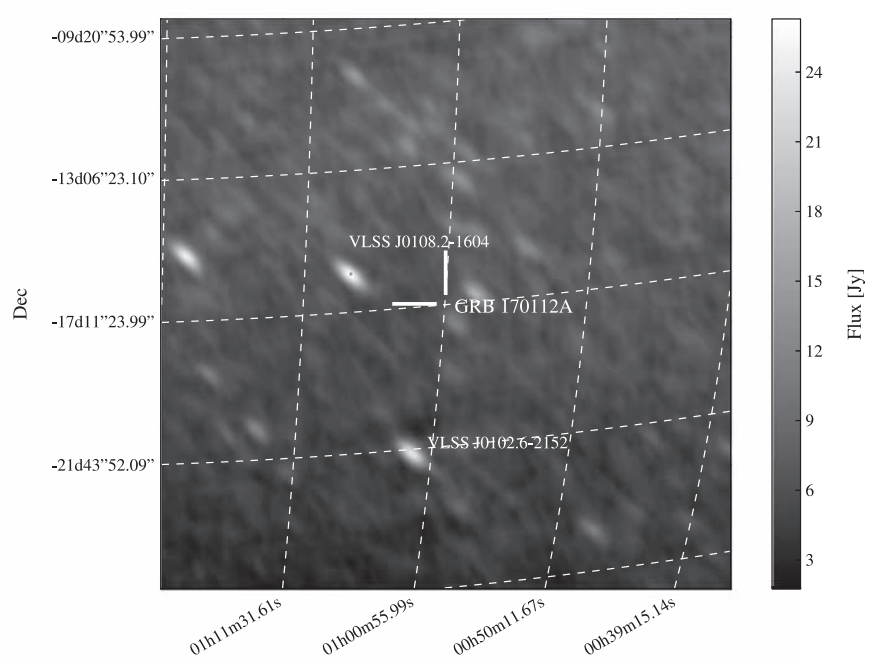

RA

(b)

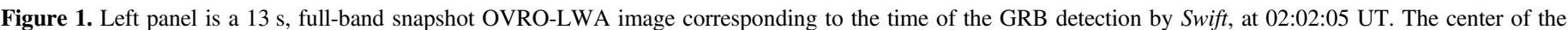

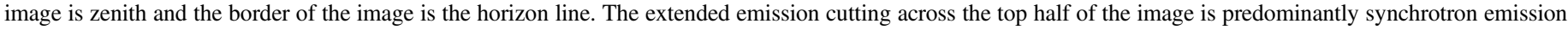

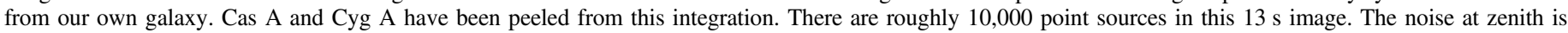

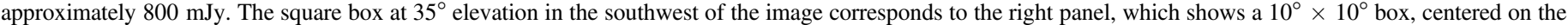
location of $170112 \mathrm{~A}$ at (R.A., decl. $)=\left(01^{\mathrm{h}} 00^{\mathrm{m}} 55^{\mathrm{s}} .7,-17^{\circ} 13^{\prime} 57^{\prime \prime} \cdot 9\right)$. VLSS J0108.2-1604 and VLSS J0102.6-2152 are also labeled.

by Lien et al. (2016) reflects that longer exposure times, and therefore longer burst durations, are needed to detect lower flux bursts. The three-burst sample with $T_{90}<0.1 \mathrm{~s}$ all having redshifts $z \lesssim 0.2$ indicates that extremely short bursts must be brighter (and thus very nearby) to be detected. However, this correlation is necessarily restricted to bursts of known redshift, and we state the caveat that bright, short bursts at high redshift may indeed be detected but are simply lacking in redshift measurements. Based on the assumptions and estimates given above, we assume an upper limit of DM $\lesssim 260 \mathrm{pc} \mathrm{cm}^{-3}$. This corresponds to a maximum dispersive delay of $\sim 1330 \mathrm{~s}$ across the full OVRO-LWA $57 \mathrm{MHz}$ bandwidth, and a maximum arrival time at the top of the band of $155 \mathrm{~s}$ after the GRB, indicating our observation covering $2 \mathrm{hr}$ post-GRB-detection is more than sufficient for capturing a coincident radio burst. The corresponding dispersion smearing across the $24 \mathrm{kHz}$ channel at the bottom of the frequency band is $\tau_{\mathrm{DM}}=8.3 \times$ $10^{3} \mathrm{~s}(\Delta \nu / \mathrm{MHz})\left(\nu_{c} / \mathrm{MHz}\right)^{-3}\left(\mathrm{DM} / \mathrm{pc} \mathrm{cm}^{-3}\right) \approx 2.5 \mathrm{~s}, \quad$ shorter than the integration time $t_{\text {int }}=13 \mathrm{~s}$ of these observations.

Figure 3 shows the undispersed dynamic spectrum at the location of GRB 170112A, formed by measuring the flux at the location of GRB 170112A in the $24 \mathrm{kHz}$ wide channel images across the full bandwidth, for every $13 \mathrm{~s}$ integration across the duration of the observation (832 integrations in total). Similar to the process by which the time series of Figure 2 was formed, we subtracted the median flux of an annulus around the GRB position of width $6 \pm 1$ synthesized beams from the flux measured at the pixel corresponding to the position of GRB 170112A in order to remove additional flux from any largescale, diffuse structure. We performed a series of dedispersion trials on the dynamic spectrum, with the spacing between DM trials set by the amount of dispersion smearing acceptable across the full bandwidth in the final, dedispersed time series. We set this equal to the integration time $t_{\text {int }}=13 \mathrm{~s}$. Following from

$$
\Delta t_{\text {smearing }}=t_{\text {int }}=4.15 \mathrm{~ms} \mathrm{DM}_{\text {step }}\left[\frac{1}{\nu_{1, \mathrm{GHz}}^{2}}-\frac{1}{\nu_{2, \mathrm{GHz}}^{2}}\right],
$$

this gives a DM step size of $\mathrm{DM}_{\text {step }}=2.5 \mathrm{pc} \mathrm{cm}^{-3}$. We search DMs spanning 0 through $1000 \mathrm{pc} \mathrm{cm}^{-3}$, despite our assumption of low DM, so as not to preclude the possibility of larger distances to the source or a significant contribution from the host galaxy or circumburst medium.

In order to search for the presence of a pulse in each of the trial DM dedispersed time series, we searched for statistically significant emission in every time series trial. Figure 4 shows the full-band dedispersed time series for every DM trial. We assume that our coarse $13 \mathrm{~s}$ integration time resolution is greater than the intrinsic pulse width (see Table 1), as well as any additional broadening due to scattering or remaining dispersion smearing due to the finite spacing of our DM trials, thus no additional smoothing is performed on the time series. Our nondetection of a radio pulse is consistent with the flat behavior of $\mathrm{S} / \mathrm{N}$ we see as a function of DM channel.

In addition to the removable effect of dispersive delay caused by the intervening plasma along the path of pulse propagation, the signal is also subject to nonremovable scatter broadening by turbulence and plasma density variations along the line of sight. The contribution to scattering from the Milky Way is negligible (a pulse broadening timescale of $\tau=0.06 \mu \mathrm{s}$ at $1 \mathrm{GHz}$ will be $\tau<t_{\text {int }}=13 \mathrm{~s}$ at $10 \mathrm{MHz}$, assuming a Kolmogorov $\nu^{-4}$ power law; Cordes \& Lazio 2002). Additional contributions to scattering are possible from the 


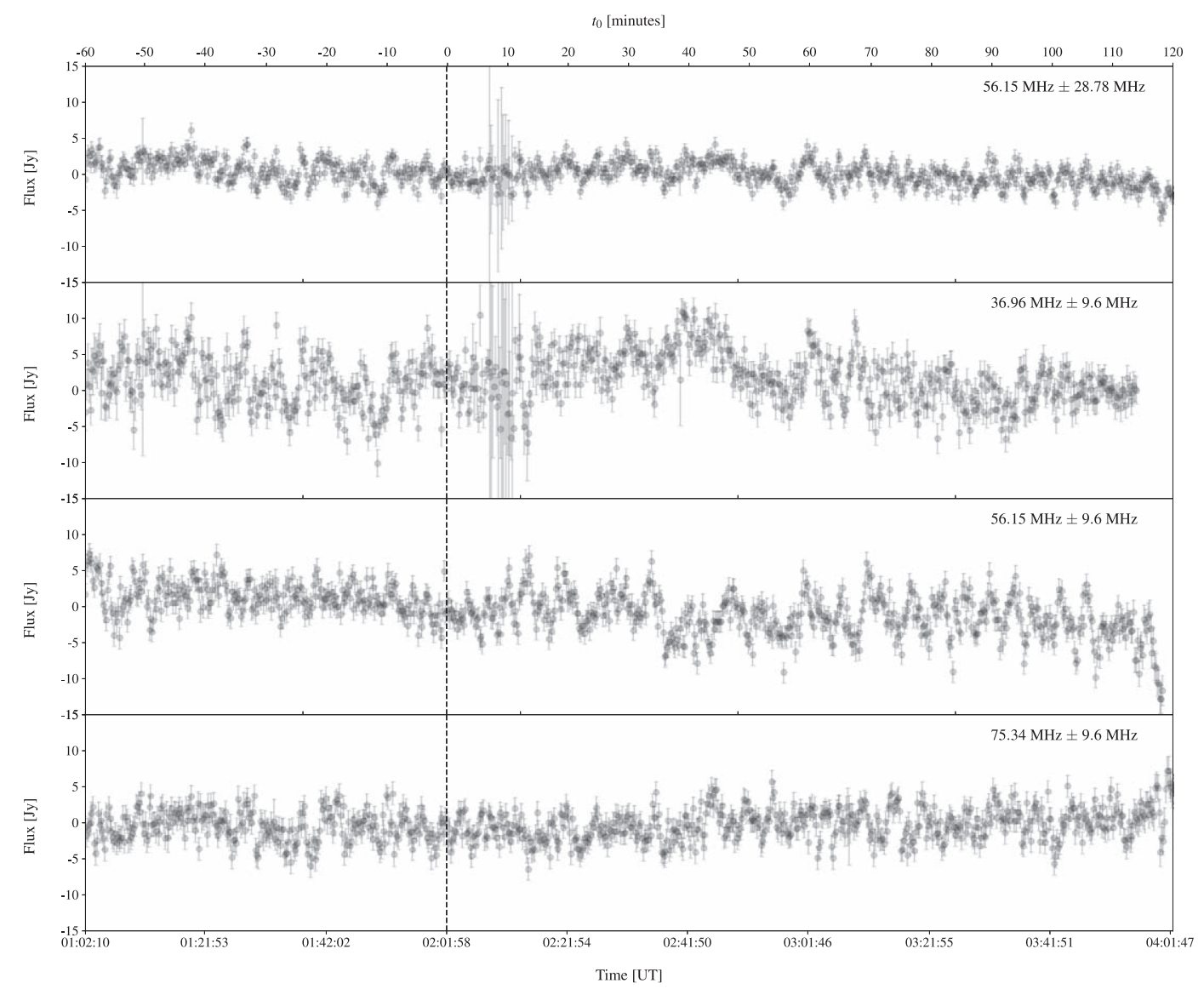

Figure 2. Flux density at the position of short GRB 170112A in each $13 \mathrm{~s}$ integration for the dedispersed time series using a DM of $260 \mathrm{pc} \mathrm{cm}^{-3}$, at $56 \mathrm{MHz}$ with the full $57 \mathrm{MHz}$ bandwidth (top), the bottom third of the band centered at $37 \mathrm{MHz}$ (second from top), the middle third of the band at $56 \mathrm{MHz}$ (third from top), and the top third of the band at $75 \mathrm{MHz}$ (bottom). The time series shows the full three-hour observation, starting one hour prior to the Swift detection of $170112 \mathrm{~A}$ at $t_{0}=0$ (dashed line) and ending two hours later. The noise in each band is $1.5 \mathrm{Jy}, 3.6 \mathrm{Jy}, 2.2 \mathrm{Jy}$, and $1.9 \mathrm{Jy}$, respectively. We detect no statistically significant emission on $13 \mathrm{~s}$ timescales indicative of prompt radio emission associated with the short GRB, and place a full band $3 \sigma$ upper flux limit of $<4.5 \mathrm{Jy}$.

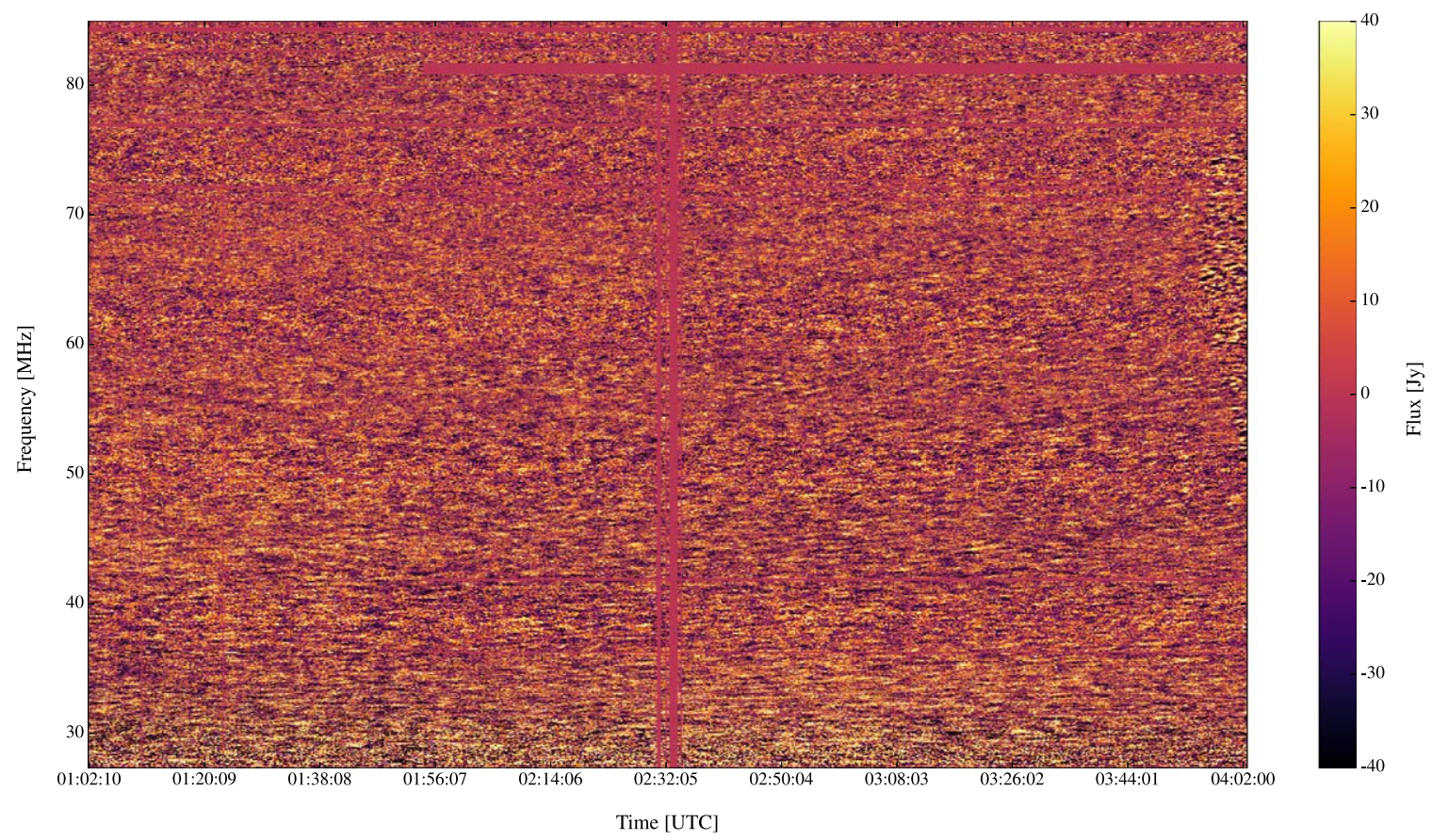

Figure 3. Dynamic spectrum measured at the position of GRB 170112A. The data span $1 \mathrm{hr}$ prior to and $2 \mathrm{hr}$ following the gamma-ray emission, at a time resolution of $13 \mathrm{~s}$. The frequency channel width is $24 \mathrm{kHz}$, with 2398 channels spanning 27.4 through $84.9 \mathrm{MHz}$. Some frequency and time bins have been flagged due to RFI (e.g., integrations surrounding 02:32:05 UTC). 

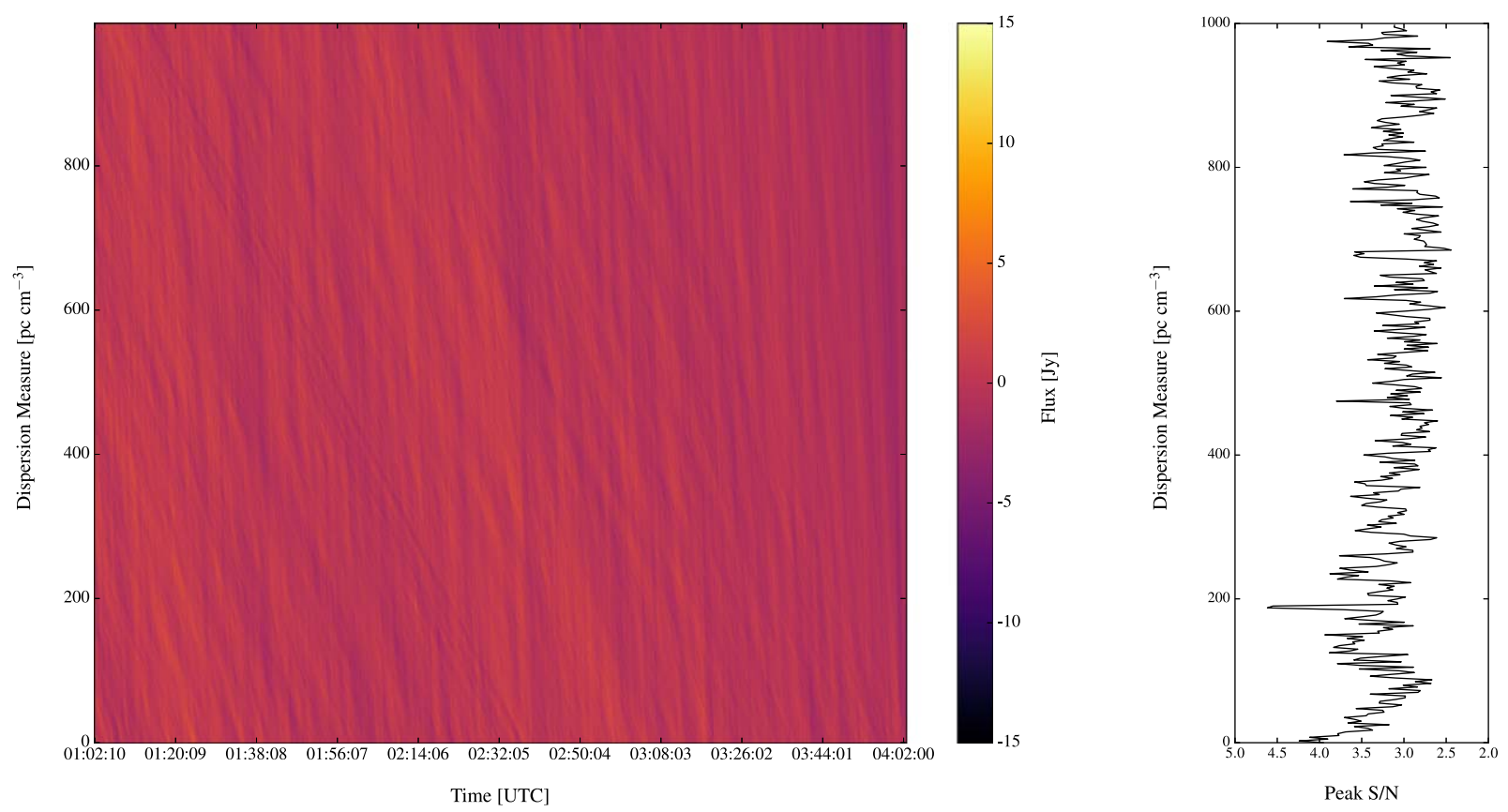

Figure 4. Dedispersed time series for every DM trial ranging from 0 to $1000 \mathrm{pc} \mathrm{cm}^{-3}$, at $2.5 \mathrm{pc} \mathrm{cm}^{-3}$ intervals. The right panel shows the peak $\mathrm{S} / \mathrm{N}$ in each dedispersed time series as a function of DM trial. No time series contains a peak S/N greater than our significance threshold. The $4.5 \sigma$ spike just below a DM of $200 \mathrm{pc} \mathrm{cm}^{-3}$ is $2 \mathrm{DM}$ channels wide, and is due to spurious unflagged frequency channels.

host galaxy, however, for the same reasons mentioned above with regards to host galaxy contribution to DM, we assume a negligible contribution from the host, given the likely significant offset of the short GRB from its host galaxy. The remaining source of scattering to be considered is the IGM. Given both the lack of correlation between DM and $\tau$ for the extragalactic fast radio bursts (FRBs) and their systematically under-scattered, relative to the Galactic DM- $\tau$ relation for pulsars, nature (Cordes et al. 2016), we assume a minimal contribution to scattering from the IGM (which we have taken as the largest contribution to the DM), and assume $\tau<t_{\text {int }}$.

\section{Discussion}

\subsection{Constraints on Models for Prompt Radio Emission}

Using our deepest limit for the prompt radio emission associated with the short GRB 170112A (57 MHz bandwidth flux density limit at a center frequency of $56 \mathrm{MHz}$ ), we can place limits on the efficiency factors (listed in column 7 of Table 1) used in the models for prompt radio emission to predict the strength of emission, as well as place an upper limit on the fractional energy released in low-frequency radio emission relative to gamma-rays in the prompt emission stage of a short GRB. Figure 5 shows the predictions for the prompt radio emission models of Hansen \& Lyutikov (2001), Pshirkov \& Postnov (2010), and Usov \& Katz (2000), associated with a 170112A-like burst, compared with our $3 \sigma$ flux density limit $S$ $(56 \mathrm{MHz})=4.5 \mathrm{Jy}$ (assuming a burst at a distance of $D=1 \mathrm{Gpc}$, and the model-predicted spectral indices $\alpha$ given in column 5 of Table 1). For the magnetized wind resulting from the magnetospheric interaction of the binary neutron stars prior to coalescence model of Hansen \& Lyutikov (2001), we place an upper limit on the efficiency of wind power conversion to radio emission of $\epsilon_{R} \lesssim 7.2 \times 10^{-3}$ (using Equation (13) of Hansen \& Lyutikov 2001 for the integrated flux density for a $13 \mathrm{~s}$ integration time that ends when the emission peaks as the neutron stars merge, and assuming a magnetic field strength of $B=10^{12} \mathrm{G}$ for the nonmagnetar component of the BNS system considered in the model). For the pulsar-like coherent radio emission powered by the rapid spin-down of the post-merger supramassive magnetar model of Pshirkov \& Postnov (2010), we place an upper limit on the efficiency with which spin-down energy is converted into coherent radio emission of $\eta \lesssim 3.8 \times 10^{-8}$, corresponding to a total energy loss rate of roughly $\dot{E}=6.1 \times 10^{51} \mathrm{erg} \mathrm{s}^{-1}$ and a power law for the spin-down power $\left(\eta \propto \dot{E}^{\gamma}\right)$ of $\gamma=-0.1$ (using Equations (6) and (7) of Pshirkov \& Postnov 2010 with a spectral index of $\alpha=-2$, and assuming the intrinsic pulse duration is $10 \mathrm{~ms}$ ). For the magnetized outflow generating coherent radio emission simultaneous to the gamma-ray emission powering the GRB model of Usov \& Katz (2000), we place an upper limit on the ratio of emitted radio to gammaray fluence of $\delta \lesssim 4.8 \times 10^{-4}$ (using Equation (14) of Usov \& Katz 2000 with a spectral index of $\alpha=1.6$, an intrinsic radio pulse duration that equals the gamma-ray $T_{90}$ of $0.06 \mathrm{~s}$, and using the measured fluence of GRB 170112A in the $15-150 \mathrm{keV}$ band of $0.13 \times 10^{-7} \mathrm{erg} \mathrm{cm}^{-2}$ ).

Finally, we can place an upper limit on the fraction of energy released in the prompt radio emission (across our $27-85 \mathrm{MHz}$ band) relative to gamma-rays. Assuming the same timescale of emission for both the prompt radio and gamma-ray emission (0.06 s for GRB 170112A); assuming a spectral index of $\alpha=-2$; using the gamma-ray fluence of $0.13 \times 10^{-7} \mathrm{erg}$ $\mathrm{cm}^{-2}$; and integrating over $27-85 \mathrm{MHz}$, the upper limit on the luminosity ratio is $L_{\text {radio }} / L_{\gamma} \lesssim 3.5 \times 10^{-6}$. 


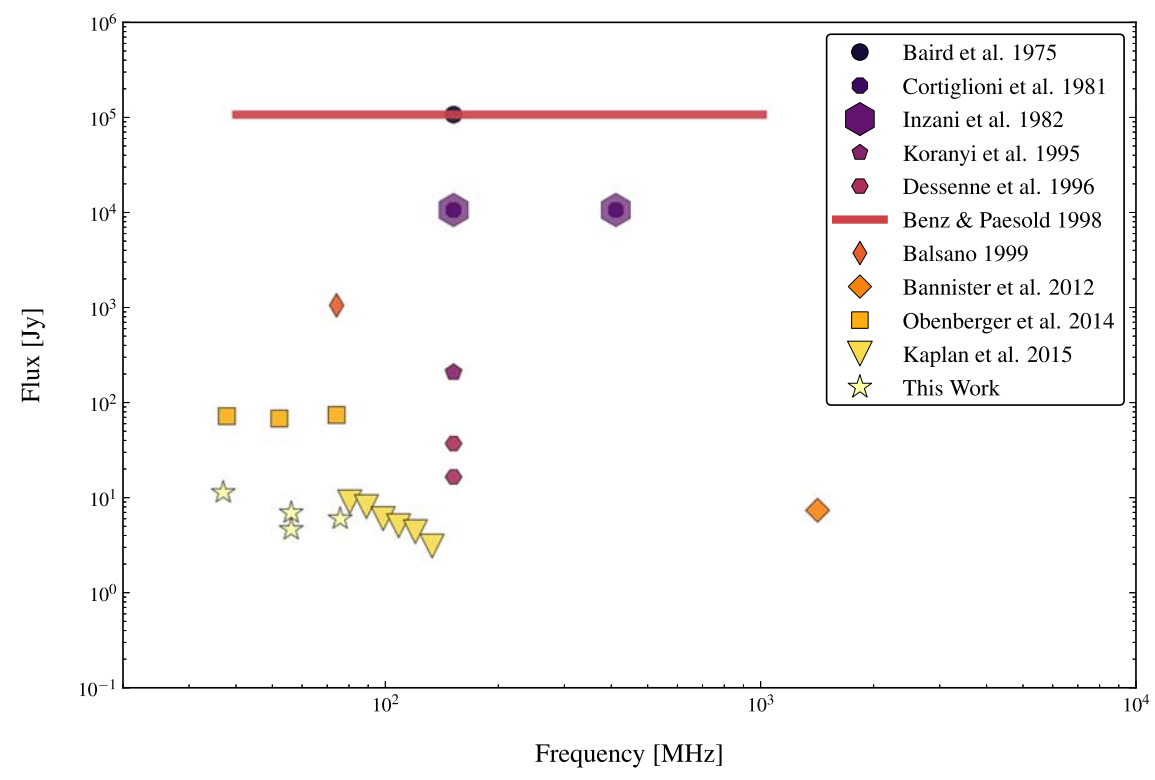

(a)

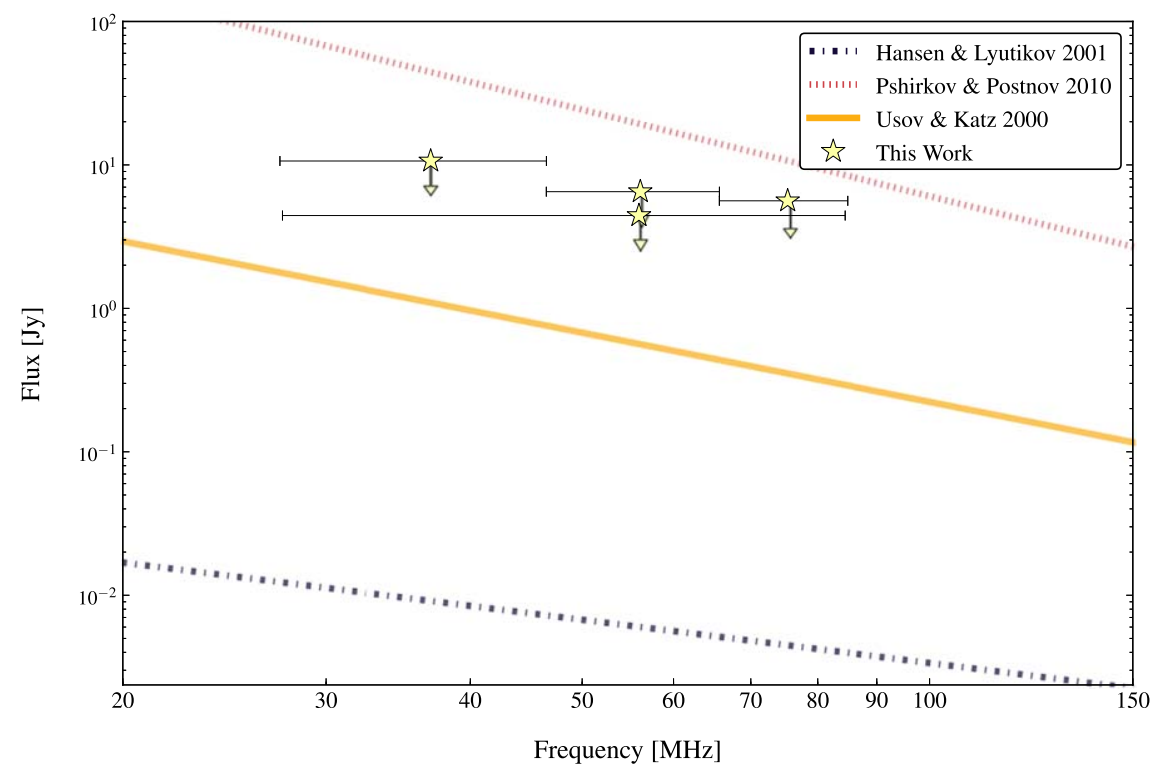

(b)

Figure 5. Top: flux density limits from all previous searches targeting prompt coherent radio emission associated with GRBs. See also Table 2. Surveys reporting limits at multiple frequencies show the corresponding number of limits in the plot. Bottom: the $3 \sigma$ flux density limits from the full $57 \mathrm{MHz}$ band and the three $19 \mathrm{MHz}$ subbands for GRB 170112A, and the model-predicted flux densities from Table 1, scaled to the $13 \mathrm{~s}$ integration time of these observations.

\subsection{Gravitational Wave Follow-up}

Verifying the existence of a prompt low-frequency radio counterpart to NS-NS(BH) merger events has taken on new significance with the detection of the binary neutron star merger GW170817 in GWs and the detection of its counterparts across the electromagnetic spectrum (Abbott et al. 2017c, 2017d; Coulter et al. 2017; Evans et al. 2017; Hallinan et al. 2017; Kasliwal et al. 2017; Troja et al. 2017). Strategies for follow-up of GW events with EM facilities are now being regularly deployed as $\mathrm{GW}$ alerts are released to partner observers and facilities, spanning the entire EM spectrum from radio to gamma-rays, and including neutrino facilities (Singer et al. 2014). Though the current models predicting prompt radio counterparts to both short and long GRBs remain very speculative, the utility of such a population for constraining important jet physics (Macquart 2007), probing the IGM (Inoue 2004), and serving as an identifiable counterpart to GW events makes the search for such events extremely valuable (e.g., Kaplan et al. 2016). The particular value of a low-frequency prompt counterpart to NS-NS(BH) mergers over counterparts at higher energies is the (1) inherently wide field of view of low-frequency facilities for rapidly and efficiently covering the entire (often $>10^{3}$ sq. deg) localization regions of GW events detected by aLIGO; (2) the time delay of 
as much as minutes, caused by the dispersion of a radio pulse by the media along the path of propagation, that allows for the pointing of telescopes; and (3) the relative dearth of variable and unrelated transient sources that could serve as false positives (unlike at optical frequencies; see, e.g., Bhalerao et al. 2017, and references therein).

The OVRO-LWA, in particular, is a uniquely powerful follow-up facility for $\mathrm{GW}$ transients. The nearly full-hemisphere ( 20,000 sq. deg.) field of view can instantaneously cover the entire $90 \%$ confidence localization region released by the two aLIGO and Virgo detectors roughly $50 \%$ of the time. The dispersive delay of a prompt radio counterpart enables timely follow-up by a number of low-frequency facilities. However, the benefits of dispersive delay become less relevant as latency between GW detection and notification of detection to partner EM facilities increases. During the first aLIGO observing run $(\mathrm{O} 1)$, the median latency between detection and notification was approximately $60 \mathrm{~s}$; however, the largest latencies extended beyond $180 \mathrm{~s}$ (Abbott et al. 2016). For GW170104, detected during the O2 run, the latency was approximately $5 \mathrm{hr}$ (Abbott et al. 2017b; LIGO Scientific Collaboration \& Virgo Collaboration 2017). However, large latency is not a limiting factor in the follow-up strategy of the OVRO-LWA, where the continuous mode of operation allows for the recovery of data from within the ring buffer, enabling contemporaneous coverage of a $\mathrm{GW}$ event and making possible the detection of any precursor radio emission, provided only that the GW alert latency is less than the duration of the OVRO-LWA buffer (which is typically $>24 \mathrm{hr}$ ).

The OVRO-LWA was observing continuously during the aLIGO O2 observing run, but was unable to search for prompt coherent emission associated with GW170817 due to the unlucky placement of the binary neutron star merger below the horizon of the OVRO-LWA at the time of the event. GW170817 was located at a distance of only $40 \mathrm{Mpc}$ (Abbott et al. 2017c), and coincident observations of a burst at this distance would have placed the model flux density predictions in Figure 5 well above the current OVRO-LWA flux density limits and allowing significant constraints on the validity of the models. The detection horizon for the Advanced LIGO and Virgo detectors during the $\mathrm{O} 3$ run to NS-NS(BH) mergers will be 220(400) Mpc (averaged over direction and inclination angle of system, Abadie et al. 2010), still well within the $1 \mathrm{Gpc}$ distance upper limit to GRB 170112A, and still closer than any short GRB of known redshift detected through prompt gammaor X-ray emission. Any burst occurring within that horizon and detected by Swift or Fermi with unknown redshift would have had to have been extremely under-luminous compared to any burst of known redshift; indeed, this was the case with GRB 170817A, which was identified in association with GW170817 but was extremely under-luminous and below the standard detection threshold of Fermi (Abbott et al. 2017a). The implication of a population of nearby merging BNS systems that do not follow the same brightness distribution or emission characteristics in their prompt high energy emission compared to their higher redshift counterparts underscores the value of an EM signature and emission mechanism that is detectable at low frequencies, for characterizing and providing an additional EM counterpart for a GW NS-NS(BH) coalescence event. The OVRO-LWA will continue the program for monitoring and triggering $\mathrm{GW}$ events during the $\mathrm{O} 3$ run, which is expected to detect between $\sim 6$ and 120 binary neutron star merger events per year at design sensitivity.

\section{Conclusion}

We have placed the most constraining upper limits to-date on prompt radio emission associated with GRBs using the OVRO-LWA to observe the field of short GRB 170112A. We searched for a pulse at the location of GRB 170112A starting $1 \mathrm{hr}$ prior to the detection of gamma-ray emission by Swift through the subsequent $2 \mathrm{hr}$, to accommodate even extremely conservative estimates for pre-merger coherent radio emission models as well as the expected dispersive delayed onset of radio emission. We see no evidence for emission in the fullband $(\Delta \nu=57 \mathrm{MHz})$ time series or following a search through the dedispersed time series for DMs ranging from 0 to $1000 \mathrm{pc}$ $\mathrm{cm}^{-3}$. We place a $1 \sigma$ flux density limit of $4.5 \mathrm{Jy}$ at $56 \mathrm{MHz}$ for $13 \mathrm{~s}$ timescales, which, assuming a nominal distance of $D=1 \mathrm{Gpc}$ to $170112 \mathrm{~A}$, allows us to place upper limits on the efficiency factors used by the models in their predictions for the luminosity of coherent radio emission associated with GRBs. We place an upper limit on the fraction of energy released in the prompt radio emission (across our $27-85 \mathrm{MHz}$ band) relative to gamma-rays of $L_{\text {radio }} / L_{\gamma} \lesssim 3.5 \times 10^{-6}$.

The contemporaneous follow-up of and sensitivity limits placed for the short GRB 170112A demonstrate the capabilities of the OVRO-LWA for targeted follow-up, particularly with regards to GW events. The OVRO-LWA is the only facility with the ability to instantaneously cover up to the full aLIGO/ Virgo localization region with zero latency, and with typical sensitivities of $\sim 800 \mathrm{mJy}$ at low frequencies. In addition, a GRB follow-up campaign with the OVRO-LWA is ongoing and will provide limits on prompt radio emission for a sample that includes both long and short GRBs, in order to encompass a wide range of potential progenitor parameters. We emphasize the caveat that the use of limits associated with GRB 170112A to constrain model parameters relies on the assumptions that any coherent radio emission will be beamed along our line of sight and that the timescale for scatter broadening of the coherent pulse is negligible compared to our integration time. These assumptions highlight the value of following up a statistical sample of GRBs, in order to place meaningful constraints on the progenitor models that do not fully rely on the aforementioned assumptions of favorable viewing.

The authors would like to thank the anonymous referee for useful and constructive comments that improved the original text of this paper. This material is based in part upon work supported by the National Science Foundation under Grant AST-1654815 and AST-1212226. G.H. acknowledges the support of the Alfred P. Sloan Foundation and the Research Corporation for Science Advancement. The OVRO-LWA project was initiated through the kind donations of Deborah Castleman and Harold Rosen.

Part of this research was carried out at the Jet Propulsion Laboratory, California Institute of Technology, under a contract with the National Aeronautics and Space Administration, including partial funding through the President's and Director's Fund Program.

Software: TTCal (Eastwood 2016), WSClean (Offringa et al. 2014b). 


\section{ORCID iDs}

Michael W. Eastwood (10 https://orcid.org/0000-0002-4731-6083 Harish K. Vedantham (iD https://orcid.org/0000-0002-0872-181X Danny C. Price (iD https://orcid.org/0000-0003-2783-1608 Frank K. Schinzel (i) https://orcid.org/0000-0001-6672-128X

\section{References}

Abadie, J., Abbott, B. P., Abbott, R., et al. 2010, CQGra, 27, 173001 Abbott, B. P., Abbott, R., Abbott, T. D., et al. 2016, ApJL, 832, L21 Abbott, B. P., Abbott, R., Abbott, T. D., et al. 2017a, ApJL, 848, L13 Abbott, B. P., Abbott, R., Abbott, T. D., et al. 2017b, PhRvL, 118, 221101 Abbott, B. P., Abbott, R., Abbott, T. D., et al. 2017c, PhRvL, 119, 161101 Abbott, B. P., Abbott, R., Abbott, T. D., et al. 2017d, ApJL, 848, L12 Baars, J. W. M., Genzel, R., Pauliny-Toth, I. I. K., \& Witzel, A. 1977, A\&A, 61, 99

Baird, G. A., Delaney, T. J., Lawless, B. G., et al. 1975, ApJL, 196, L11 Balsano, R. J. 1999, PhD thesis, Princeton Univ.

Bannister, K. W., Murphy, T., Gaensler, B. M., \& Reynolds, J. E. 2012, ApJ, 757, 38

Belczynski, K., Perna, R., Bulik, T., et al. 2006, ApJ, 648, 1110

Benz, A. O., \& Paesold, G. 1998, A\&A, 329, 61

Berger, E. 2009, ApJ, 690, 231

Berger, E., Price, P. A., Cenko, S. B., et al. 2005, Natur, 438, 988

Bhalerao, V., Kasliwal, M. M., Bhattacharya, D., et al. 2017, ApJ, 845, 152

Bloom, J. S., Prochaska, J. X., Pooley, D., et al. 2006, ApJ, 638, 354

Bloom, J. S., Sigurdsson, S., \& Pols, O. R. 1999, MNRAS, 305, 763

Bostancı, Z. F., Kaneko, Y., \& Göğüş, E. 2013, MNRAS, 428, 1623

Briggs, D. S. 1995, PhD thesis, New Mexico Institute of Mining and Technology

Cordes, J. M., \& Lazio, T. J. W. 2002, arXiv:astro-ph/0207156

Cordes, J. M., \& McLaughlin, M. A. 2003, ApJ, 596, 1142

Cordes, J. M., Wharton, R. S., Spitler, L. G., Chatterjee, S., \& Wasserman, I. 2016, ApJ, submitted (arXiv:1605.05890)

Cortiglioni, S., Mandolesi, N., Morigi, G., et al. 1981, Ap\&SS, 75, 153

Costa, E., Frontera, F., Heise, J., et al. 1997, Natur, 387, 783

Coulter, D. A., Foley, R. J., Kilpatrick, C. D., et al. 2017, Sci, 358, 1556

D'Ai, A., Melandri, A., D'Avanzo, P., et al. 2017, GCN, 20440

D'Avanzo, P., Fugazza, D., Covino, S., \& Melandri, A. 2017, GCN, 20438

Dessenne, C. A.-C., Green, D. A., Warner, P. J., et al. 1996, MNRAS, 281, 977

Duez, M. D., Liu, Y. T., Shapiro, S. L., Shibata, M., \& Stephens, B. C. 2006, PhRvD, 73, 104015

Eastwood, M. W. 2016, TTCal, v0.3.0, Zenodo, doi:10.5281/zenodo.1049160 Eastwood, M. W., Anderson, M. M., Monroe, R. M., et al. 2018, AJ, 156, 32 Ellingson, S. W., Craig, J., Dowell, J., Taylor, G. B., \& Helmboldt, J. F. 2013, arXiv: 1307.0697

Evans, P. A., Cenko, S. B., Kennea, J. A., et al. 2017, Sci, 358, 1565

Fong, W., \& Berger, E. 2013, ApJ, 776, 18

Frail, D. A., Kulkarni, S. R., Nicastro, L., Feroci, M., \& Taylor, G. B. 1997, Natur, 389, 261

Fruchter, A. S., Levan, A. J., Strolger, L., et al. 2006, Natur, 441, 463

Gehrels, N., Chincarini, G., Giommi, P., et al. 2004, ApJ, 611, 1005

Goldreich, P., \& Reisenegger, A. 1992, ApJ, 395, 250

Granot, J., \& van der Horst, A. J. 2014, PASA, 31, e008

Hallinan, G., Corsi, A., Mooley, K. P., et al. 2017, Sci, 358, 1579

Hansen, B. M. S., \& Lyutikov, M. 2001, MNRAS, 322, 695
Inoue, S. 2004, MNRAS, 348, 999

Inzani, P., Sironi, G., Mandolesi, N., \& Morigi, G. 1982, in AIP Conf. Ser. 77, Gamma Ray Transients and Related Astrophysical Phenomena, ed. R. E. Lingenfelter, H. S. Hudson, \& D. M. Worrall (Melville, NY: AIP), 79 Kaplan, D. L., Murphy, T., Rowlinson, A., et al. 2016, PASA, 33, e050 Kaplan, D. L., Rowlinson, A., Bannister, K. W., et al. 2015, ApJL, 814, L25 Kasliwal, M. M., Nakar, E., Singer, L. P., et al. 2017, Sci, 358, 1559 Klebesadel, R. W., Strong, I. B., \& Olson, R. A. 1973, ApJL, 182, L85

Kocz, J., Greenhill, L. J., Barsdell, B. R., et al. 2015, JAI, 4, 1550003

Koranyi, D. M., Green, D. A., Warner, P. J., Waldram, E. M., \& Palmer, D. M 1995, MNRAS, 276, L13

Kouveliotou, C., Meegan, C. A., Fishman, G. J., et al. 1993, ApJL, 413, L101 Krimm, H. A., Holland, S. T., Corbet, R. H. D., et al. 2013, ApJS, 209, 14 Lane, W. M., Cotton, W. D., van Velzen, S., et al. 2014, MNRAS, 440, 327 Li, L.-X., \& Paczyński, B. 1998, ApJL, 507, L59

Lien, A., Sakamoto, T., Barthelmy, S. D., et al. 2016, ApJ, 829, 7

Lien, A. Y., Barthelmy, S. D., Cummings, J. R., et al. 2017, GCN, 20443

LIGO Scientific Collaboration and Virgo Collaboration 2017, GCN, 20364, https://gcn.gsfc.nasa.gov/gcn3/20364.gcn3

Lyutikov, M. 2009, arXiv:0911.0349

Lyutikov, M. 2013, ApJ, 768, 63

Macquart, J.-P. 2007, ApJL, 658, L1

Mazaeva, E., Pozanenko, A., Inasaridze, R., et al. 2017, GCN, 20472

McMullin, J. P., Waters, B., Schiebel, D., Young, W., \& Golap, K. 2007, in ASP Conf. Ser. 376, Astronomical Data Analysis Software and Systems XVI, ed. R. A. Shaw, F. Hill, \& D. J. Bell (San Francisco, CA: ASP), 127

Metzger, B. D., Quataert, E., \& Thompson, T. A. 2008, MNRAS, 385, 1455

Metzger, B. D., Thompson, T. A., \& Quataert, E. 2018, ApJ, 856, 101

Mingo, B., Burrows, D. N., Gronwall, C., et al. 2017, GCN, 20436

Moortgat, J., \& Kuijpers, J. 2005, in Proc. of the 22nd Texas Symp. Relativistic Astrophysics, ed. P. Chen et al. (Stanford, CA: United States Department of Energy), 326

Narayan, R., Paczynski, B., \& Piran, T. 1992, ApJL, 395, L83

Obenberger, K. S., Hartman, J. M., Taylor, G. B., et al. 2014, ApJ, 785, 27

Obenberger, K. S., Taylor, G. B., Hartman, J. M., et al. 2015, JAI, 4, 1550004

Offringa, A. R., McKinley, B., Hurley-Walker, N., et al. 2014a, MNRAS, 444,606

Offringa, A. R., McKinley, B., Hurley-Walker, N., et al. 2014b, WSClean: Widefield Interferometric Imager, Astrophysics Source Code Library, ascl:1408.023

Palmer, D. M. 1993, ApJL, 417, L25

Price, D. C., Greenhill, L. J., Fialkov, A., et al. 2018, MNRAS, 478, 4193

Pshirkov, M. S., \& Postnov, K. A. 2010, Ap\&SS, 330, 13

Sagiv, A., \& Waxman, E. 2002, ApJ, 574, 861

Savaglio, S., Glazebrook, K., \& Le Borgne, D. 2009, ApJ, 691, 182

Siegel, M. H., \& Mingo, B. 2017, GCN, 20451

Singer, L. P., Price, L. R., Farr, B., et al. 2014, ApJ, 795, 105

Soderberg, A. M., Berger, E., Kasliwal, M., et al. 2006, ApJ, 650, 261

Tanvir, N. R., Levan, A. J., Fruchter, A. S., et al. 2013, Natur, 500, 547

Tingay, S. J., Goeke, R., Bowman, J. D., et al. 2013, PASA, 30, e007

Troja, E., Piro, L., van Eerten, H., et al. 2017, Natur, 551, 71

Usov, V. V., \& Katz, J. I. 2000, A\&A, 364, 655

van Paradijs, J., Groot, P. J., Galama, T., et al. 1997, Natur, 386, 686

Woosley, S. E., \& Bloom, J. S. 2006, ARA\&A, 44, 507

Yang, B., Jin, Z.-P., Li, X., et al. 2015, NatCo, 6, 7323

Zheng, Z., Ofek, E. O., Kulkarni, S. R., Neill, J. D., \& Juric, M. 2014, ApJ, 797,71 